International Journal of Pure and Applied Mathematics

Volume 86 No. 5 2013, 849-857

ISSN: 1311-8080 (printed version); ISSN: 1314-3395 (on-line version)

url: http://www.ijpam.eu

doi: http://dx.doi.org/10.12732/ijpam.v86i5.8

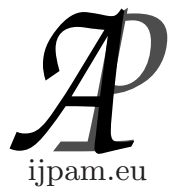

\title{
EXISTENCE AND UNIQUENESS OF SELF-SIMILAR SOLUTIONS OF A NONHOMOGENEOUS EQUATION
}

\author{
Badia Hamri ${ }^{1}$, Abdelilah Gmira ${ }^{2}$, Youssef Jabri ${ }^{3} \S$ \\ ${ }^{1,2}$ Department of Mathematics \\ Faculty of Science \\ University Abdelmalek Essaâdi \\ B.P. 2121, Tétouan, MOROCCO \\ ${ }^{3}$ Department of Mathematics \\ National School of Applied Sciences \\ University Mohammed I \\ B.P. 669 , Oujda, MOROCCO
}

Abstract: In this paper, we shall prove the existence and uniqueness of radial solutions for the nonhomogeneous elliptic equation

$$
\operatorname{div}\left(|\nabla u|^{p-2} \nabla u\right)+\beta x \nabla u+\alpha u+|x|^{l}|u|^{q-1} u=0, \quad x \in \mathbb{R}^{N},
$$

These solutions are related to self-similar solutions of the degenerate parabolic equation

$$
v_{t}=\operatorname{div}\left(|\nabla v|^{p-2} \nabla v\right)+|x|^{l}|v|^{q-1} v, \quad(t, x) \in(0,+\infty) \times \mathbb{R}^{N} .
$$

where $p>2, q \geq 1, N \geq 1,-p<l,-N<l$.

AMS Subject Classification: 35K55, 35K65

Key Words: elliptic problem, parabolic problem, nonhomogeneous equation, self-similar solution, existence, uniqueness

\section{Introduction}

In this paper, we analyse the self-similar solutions of a nonhomogeneous degen-

Received: May 15, 2013

(C) 2013 Academic Publications, Ltd.

$\S_{\text {Correspondence author }}$ 
erate parabolic equation

$$
w_{t}=\Delta_{p} w+|x|^{l}|w|^{q-1} w, \text { in } \mathbb{R}^{\mathbb{N}} \times(0,+\infty)
$$

where $p>2, q \geq 1, N \geq 1,-p<l<0,-N<l<0$.

As usual, $\nabla U$ denotes the gradient of $U$, and $\Delta_{p} U=\operatorname{div}\left(|\nabla U|^{p-2} \nabla U\right)$ is the $p$-Laplacian operator.

If we look for self-similar solutions of the form

$$
w(x, t)=t^{-\alpha} U\left(t^{-\beta} x\right), \quad \text { in } \mathbb{R}^{\mathbb{N}} \times(0,+\infty),
$$

then the profile $U$ must satisfy the nonhomogeneous elliptic equation

$$
\Delta_{p} U+\beta x \nabla U+\alpha U+|x|^{l}|U|^{q-1} U=0, \text { for } x \in \mathbb{R}^{\mathbb{N}},
$$

where the scaling factors are given by

$$
\alpha=\frac{p+l}{p(q-1)+l(p-2)}, \quad \beta=\frac{q-p+1}{p(q-1)+l(p-2)},
$$

with $l \neq-\frac{p(q-1)}{p-2}$.

It is worth mentioning that several recent papers have been devoted to the study of equation (1.3) when $l=0$ and $p=2$, cf. e.g; [5, 6, 7, 8, 10]. Quite recently, in [4] the case $p=2$ and $-2<l<0$ was treated. Note also when $l=0$ and $p>2$ our equation was investigated in [2] and [9]. The present paper is devoted to the case $p>2,-p<l<0,-N<l<0$ and, $\alpha$ and $\beta$ are real parameters. More precisely, the main purpose of this paper is to study existence and uniqueness of radially-symmetric solutions; which are functions $U(y)=u(|y|)=u(r)$ where $u: \mathbb{R}^{+} \rightarrow \mathbb{R}$ satisfies the following O.D.E

$$
\left(\left|u^{\prime}\right|^{p-2} u^{\prime}\right)^{\prime}+\frac{N-1}{r}\left|u^{\prime}\right|^{p-2} u^{\prime}+\alpha u+\beta r u^{\prime}+r^{l}|u|^{q-1} u=0, r>0,
$$

the prime denotes the differentiation with respect to $r$.

In order to study equation (1.5) we shall use the shooting method, we then consider the following Cauchy problem $(P)$

$$
\text { (P) }\left\{\begin{array}{l}
\left(\left|u^{\prime}\right|^{p-2} u^{\prime}\right)^{\prime}+\frac{N-1}{r}\left|u^{\prime}\right|^{p-2} u^{\prime}+\alpha u+\beta r u^{\prime}+r^{l}|u|^{q-1} u=0 \\
u(0)=a
\end{array}\right.
$$

where $p>2, q \geq 1, N \geq 1,0>l>-p$ and $0>l>-N$, and $\alpha, \beta, a$ are real parameters. 


\section{Existence and Uniqueness of Solutions}

In this section we investigate the existence and uniqueness of a solution $u$ of the problem $(P)$.

By a solution of $(P)$ on some interval $[0, R[$ we mean a function $u \in$ $C^{0}\left(\left[0, R[) \cap C^{1}(] 0, R[)\right.\right.$ such that $\left|u^{\prime}\right|^{p-2} u^{\prime} \in C^{1}(] 0, R[)$ and which satisfies (1.6) on the open interval ]0, $R$ [, with $\lim _{r \rightarrow 0} r^{N-1}\left|u^{\prime}\right|^{p-2} u^{\prime}=0$.

To emphasize the dependence of $u$ on the parameters $\alpha, \beta$ and $a$, we sometimes denote a solution of $(P)$ by $u(., a, \alpha, \beta)$.

We start with a local existence and uniqueness result.

Proposition 2.1. Assume $p>2, q \geq 1, N \geq 1,-p<l<0,-N<l$ and $\alpha, \beta \in \mathbb{R}^{*}$. Then for any $a \in \mathbb{R}^{*}$, there exists a constant $R(a)>0$ such that the problem $(P)$ has a unique maximal solution $u$ defined in $[0, R(a)[$.

Remark 2.1. Note that equation (1.6) can be written as in the following system

$$
(S)\left\{\begin{array}{l}
u^{\prime}=|v|^{-\frac{p-2}{p-1}} v, \\
v^{\prime}=-\frac{N-1}{r} v-\beta r|v|^{-\frac{p-2}{p-1}} v-\alpha u-r^{l}|u|^{q-1} u .
\end{array}\right.
$$

Let $r_{0}>0, \mu \in \mathbb{R}$ and $\eta \in \mathbb{R}^{*}$ such that

$$
\left\{\begin{array}{l}
u\left(r_{0}\right)=\mu \\
v\left(r_{0}\right)=\eta
\end{array}\right.
$$

As $(r, u, v) \mapsto\left(r,|v|^{-\frac{p-2}{p-1}} v,-\frac{N-1}{r} v-\beta r|v|^{-\frac{p-2}{p-1}} v-\alpha u-r^{l}|u|^{q-1} u\right)$ is locally Lipschitz continuous function in the set $\left\{(r, u, v) \in \mathbb{R}_{+}^{*} \times \mathbb{R} \times \mathbb{R}^{*}\right\}$, we deduce (from the theory of O.D.E [1]) the local existence and uniqueness solution of $(2.1)-(2.2)$.

In spite of the degenerescence of $(P)$ at $r=0$, the Banach fixed point theorem ensures the local existence and uniqueness. We prove that this solution is global indeed.

Let $u$ be a solution of problem $(P)$ in some interval $[0, R[$; then if we multiply (1.6) by $r^{N-1}$ and integrate twice from 0 to $r(0<r<R)$ we get

$$
u(r)=a-\int_{0}^{r} \Psi\left\{f_{u}(s)\right\} d s,
$$

where

$$
f_{u}(s)=\beta s u(s)
$$




$$
+s^{1-N}\left[(\alpha-\beta N) \int_{0}^{s} \sigma^{N-1} u(\sigma) d \sigma+\int_{0}^{s} \sigma^{l+N-1}|u|^{q-1} u(\sigma) d \sigma\right]
$$

and

$$
\Psi(s)=|s|^{-(p-2) /(p-1)} s \quad \text { for any } s \in \mathbb{R}^{*} .
$$

Let us introduce some notations. For any $0<M<a$, we denote

$$
E_{M, a}=\left\{u \in C^{0}\left(\left[0, r_{a}\right]\right) ;\|u-a\|_{E_{M, a}} \leq M\right\}
$$

where $C^{0}\left(\left[0, r_{a}\right]\right)$ is the Banach space of continuous functions on $\left[0, r_{a}\right]$ where

$$
r_{a}=\min \left\{r_{i}, i=1,2,3\right\}
$$

The reals $r_{i}$ are given explicitly by

$$
\begin{gathered}
r_{1}=k_{2}^{-\frac{1}{p+l}}\left[\frac{p+l}{p-1} M\right]^{\frac{p-1}{p+l}}, \\
r_{2}=K_{1}^{\frac{p-2}{l+p}}\left[\frac{p+l}{2 q} \frac{l+N}{(M+a)^{q-1}}\right]^{\frac{p-1}{p+l}}, \\
r_{3}=k_{1}^{\frac{p-2}{p-l(p-2)}}\left[\frac{p-l(p-2)}{2}\left(|\beta|+\frac{|\alpha-N \beta|}{N}\right)^{-1}\right]^{\frac{p-1}{p-l(p-2)}},
\end{gathered}
$$

with

$$
K_{1}=\frac{(a-M)^{q}}{2(l+N)} \text { and } K_{2}=2 \frac{(a+M)^{q}}{(l+N)} .
$$

The following result is the keystone of the proof of the existence.

Lemma 2.1. Let $a \in \mathbb{R}_{*}^{+}$and $0<M<a$. For each $v \in E_{M, a}$, the function $f_{v}$ given by (2.4) satisfies

$$
K_{1} s^{l+1}<f_{v}(s) \leq K_{2} s^{l+1}, \forall 0 \leq s \leq r_{0},
$$

where $r_{a}$ as in the formula (2.7) and $K_{1}$ and $K_{2}$ are given by (2.11).

Proof. The idea is to limit the function $f_{v}(r)$ with two expressions having the same sign. These expressions depend strongly on the sign of $\beta$ and $\alpha-N \beta$. For this purpose we assume $\beta \geq 0$ and $\alpha-N \beta \geq 0$, (the reasoning in the others three cases is similar). Using the definition of $E_{M, a}$, the following estimates hold for any $v \in E_{M, a}$ : 


$$
\begin{aligned}
\left\{\left(\frac{\alpha}{N}-\beta\right)+\frac{(a-M)^{q-1}}{l+N} s^{l}\right\}(a-M) s \leq g(s) & \\
& \leq\left\{\left(\frac{\alpha}{N}-\beta\right)+\frac{(a+M)^{q-1}}{l+N} s^{l}\right\}(a+M) s,
\end{aligned}
$$

where

$$
g(s)=s^{1-N}\left[(\alpha-\beta N) \int_{0}^{s} \sigma^{N-1} v(\sigma) d \sigma+\int_{0}^{s} \sigma^{l+N-1}|v|^{q-1} v(\sigma) d \sigma\right] .
$$

Hence if we chose $s$ small enough we get

$$
\frac{(a-M)^{q}}{2(l+N)} s^{l+1} \leq f_{v}(s) \leq 2 \frac{(a+M)^{q}}{(l+N)} s^{l+1}
$$

Then the lemma follows.

Now we are able to prove the proposition.

Proof. The proof is divided into two steps.

Step 1: Local Existence and Uniqueness. For the set $E_{M, a}$ given by (2.6), consider the mapping $T$ defined on $E_{M, a}$ by

$$
T(v)(r)=a-\int_{0}^{r} \Psi\left(f_{v}\right)(s) d s
$$

where $\Psi$ and $f_{v}$ are given by (2.4) and (2.5). Using Lemma 2.1 we deduce

$$
|T(v)(r)-a| \leq \frac{p-1}{l+p} K_{2}^{\frac{1}{p-1}} r^{\frac{l+p}{p-1}} .
$$

From the choice of (2.7) we deduce that $T$ maps $E_{M_{, a}}$ into itself.

Now we assert that $T$ is a contraction. In fact let $v, w \in E_{M, a}$ and $r \in\left[0, r_{a}\right]$, then

$$
\begin{aligned}
& |T(v)(r)-T(w)(r)| \\
& \quad \leq \int_{0}^{r}\left|\Psi\left(f_{v}(s)\right)-\Psi\left(f_{w}(s)\right)\right| d s, \text { for any } r \in\left[0, r_{a}\right] .
\end{aligned}
$$

Set

$$
\Phi(s)=\min \left(\left|f_{v}(s)\right|,\left|f_{w}(s)\right|\right) \text { for any } s \in\left[0, r_{a}\right],
$$

then

$$
|T(v)(r)-T(w)(r)| \leq \int_{0}^{r} \frac{\Psi(\Phi(s))}{\Phi(s)}\left|\left(f_{v}-f_{w}\right)(s)\right| d s .
$$


Since the estimate (2.12) holds for $f_{v}$ and also for $f_{w}$, we get

$$
\begin{array}{r}
\left|\Psi\left(f_{v}(s)\right)-\Psi\left(f_{w}(s)\right)\right| \leq \frac{1}{p-1}\left|K_{1} s^{l+1}\right|^{-\frac{p-2}{p-1}}\left|f_{v}(s)-f_{w}(s)\right| \\
\quad \text { for any } s \in\left[0, r_{a}\right]
\end{array}
$$

But (2.11) and the definition of the space $E_{M, a}$, imply that the following estimate holds true

$$
\left|f_{v}(s)-f_{w}(s)\right| \leq\left[\left(|\beta|+\frac{|\alpha-\beta N|}{N}\right) s+q \frac{(M+a)^{q-1}}{l+N} s^{l+1}\right]\|v-w\|_{0},
$$

for any $s \in\left[0, r_{a}\right]$. Putting together (2.20), (2.21) and (2.22) we get

$$
\begin{aligned}
& |T(v)(r)-T(w)(r)| \leq \frac{1}{l+p} K_{1}^{-\frac{p-2}{p-1}}\left[q \frac{(M+|a|)^{q-1}}{l+N} r^{\frac{l+p}{p-1}}\right. \\
& \left.+\frac{1}{p-l(p-2)}\left(|\beta|+\frac{|\alpha-\beta N|}{N}\right) r^{\frac{p-l(p-2)}{p-1}}\right]\|v-w\|_{E_{M, a}}
\end{aligned}
$$

for any $0<r \leq r_{a}$.

From the choice of $r_{a}$, we conclude that $T$ is a contraction; and then the Banach contraction theorem implies that there exists a unique function $u$ solving the problem $(P)$ in $\left[0, r_{a}\right]$.

Note that as $u$ is continuous, as given by (2.3). We deduce easily that $u \in C^{1}(] 0, r_{a}[)$ and also $\left|u^{\prime}\right|^{p-2} u^{\prime} \in C^{1}(] 0, r_{a}[)$.

Step 2. $\lim _{r \rightarrow 0} r^{N-1}\left|u^{\prime}\right|^{p-2} u^{\prime}=0$.

Using again the implicit formula given by (2.7) we deduce

$$
\begin{aligned}
\left|u^{\prime}\right|^{p-2} u^{\prime}(r)=\beta r u(r)+r^{1-N}[(\alpha-\beta N) & \int_{0}^{r} s^{N-1} u(s) d s \\
& \left.+\int_{0}^{r} s^{l+N-1}|u|^{q-1} u(s) d s\right]
\end{aligned}
$$

for any $0<r \leq r_{a}$. As $l>-N$ we obtain $\lim _{r \rightarrow 0} r^{N-1}\left|u^{\prime}\right|^{p-2} u^{\prime}=0$, which completes the proof.

Remark 2.2. The following holds true:

i) It is easy to see that the function $f_{v}$ has the following behavior

$$
f_{v}(r) \simeq a\left\{\frac{\alpha}{N}+\frac{1}{l+N} a^{q-1} r^{l}\right\} r
$$


when $r$ tends towards 0 . We deduce that if $-N<l<0$ and $-p<l<0$ then for any $a>0$ the solution $u$ starts decreasing independently of sign of $\alpha$ and $\beta$. Moreover, (1.6) implies that if $\alpha>0$ and $\beta>0$ the solution $u$ is strictly decreasing until it reaches the axis of $x$.

ii) Proposition 2.1 is also valid for $l \geq 0$, if we replace the power $l+1$ in formula (2.15) by 1.

iii) Note that $u(., a, \alpha, \beta)=-u(.,-a, \alpha, \beta)$.

Proposition 2.2. Let $\alpha, \beta \in \mathbb{R}, q>1, p>2,-p<l<0$ and $-N<l$. Then for any $a>0$, the solution $u$ of the problem $(P)$ is global.

Proof. Let $[0, R(a)$ [ be the maximum interval of existence. Integrating equation (1.6) on $] r_{0}, r\left[\right.$ for some $0<r_{0}<r<R(a)$, we get

$$
\begin{aligned}
& \frac{p-1}{p}\left|u^{\prime}\right|^{p}(r)-\frac{p-1}{p}\left|u^{\prime}\right|^{p}\left(r_{0}\right)-\frac{\alpha}{2} u^{2}\left(r_{0}\right)-\frac{r_{0}^{l} u^{q+1}\left(r_{0}\right)}{q+1}= \\
& +u^{2}(r)\left[\frac{\alpha}{2}+\frac{r^{l}|u(r)|^{q-1}}{q+1}\right]-(N-1) \int_{r_{0}}^{r} \frac{1}{s}\left|u^{\prime}(s)\right|^{p} d s \\
& -\beta \int_{r_{0}}^{r} s\left(u^{\prime}\right)^{2}(s) d s+\frac{l}{q+1} \int_{r_{0}}^{r} s^{l-1}|u(s)|^{q+1} d s
\end{aligned}
$$

Assume $R(a)$ is finite. Then, the functions $u(r)$ and $u^{\prime}(r)$ go to $\infty$ when $r$ goes to $R(a)$. As $l<0$ we claim that the right hand side of $(2.26)$ is strictly negative for $r$ close to $R(a)$. This is obvious for $\beta \geq 0$. On the other hand, when $\beta<0$ we use the following estimate

$$
\int_{r_{0}}^{r} s\left(u^{\prime}\right)^{2}(s) d s \leq\left[\frac{r^{2}}{2}-\frac{r_{0}^{2}}{2}\right]^{\frac{p-2}{p}}\left[\int_{r_{0}}^{r} \frac{1}{s}\left|u^{\prime}(s)\right|^{p} d s\right]^{\frac{2}{p}}
$$

to deduce that

$$
\begin{aligned}
& -(N-1) \int_{r_{0}}^{r} \frac{1}{s}\left|u^{\prime}(s)\right|^{p} d s-\beta \int_{r_{0}}^{r} s\left(u^{\prime}\right)^{2}(s) d s \leq \\
& {\left[\int_{r_{0}}^{r} \frac{\left|u^{\prime}(s)\right|^{p}}{s} d s\right]^{\frac{2}{p}}\left\{|\beta|\left(\frac{r^{2}}{2}-\frac{r_{0}^{2}}{2}\right)^{\frac{p-2}{p}}-(N-1)\left[\int_{r_{0}}^{r} \frac{\left|u^{\prime}(s)\right|^{p}}{s} d s\right]^{\frac{p-2}{p}}\right\},}
\end{aligned}
$$

which implies that the right hand side of (2.26) is strictly negative, for $r$ close to $R(a)$, while the expression $\frac{\alpha}{2}+\frac{r^{l} \mid u(r) q^{q-1}}{q+1}$ goes to $+\infty$ when $r$ tends to $R(a)$, and so the left side of (2.26) is strictly positive. This is a contradiction. Thereby $[0, R(a)[=[0, \infty[$. 
Remark 2.3. Note that if $\alpha>0, \beta>0$ and $-p<l<0$ and $-N<l$ the global existence can be shown easily. In fact consider the energy function defined by

$$
E(r)=\frac{p-1}{p}\left|u^{\prime}\right|^{p}+\frac{\alpha}{2} u^{2}+r^{l} \frac{|u|^{q+1}}{q+1}
$$

According to equation (1.6), E satisfies

$$
E^{\prime}(r)=-\left\{\frac{N-1}{r}\left|u^{\prime}\right|^{p}+\beta r u^{\prime 2}-\frac{l}{q+1} r^{l-1}|u|^{q+1}\right\} .
$$

As $l<0$, the energy is strictly decreasing and then the maximal existence interval is $[0, \infty[$.

\section{References}

[1] H. Amann, Ordinary Differential Equations, Walter de Gruyter Inc., Germany, 1996.

[2] B. Benyounes, A. Gmira, On the radial solutions of degenerate quasilinear elliptic equation, Annales de la Faculté des Sciences de Toulouse, 8, No. 3 (1999), 411-438.

[3] M.F. Bidaut-Véron , S. Pohozaev, Nonexistence results and estimates for some nonlinear elliptic problems, J. Anal. Math., 84 (2001), 1-49.

[4] S. Filippas, A. Tertikas, On similarity solutions of a heat equation with a nonhomogeneous nonlilearity, J. Diff. Equ., 165 (2000), 468-492.

[5] A. Haraux, F.B. Weissler, Non-uniqueness for a semilinear initial value problem, Indiana Univ. Math. J., 31 (1982) 167-189.

[6] M. Hirose, E. Yanagida, Global structure of self-similar solutions for semilinear heat equations, J. Diff. Equ., 163 (2000), 407-428.

[7] L.A. Peletier, D. Terman, F.B. Weissler, On the equation $\triangle u+\frac{1}{2} x . \nabla u+$ $f(u)=0$, Archive for Rational Mechanics and Analysis , 94 (1986), 83-99.

[8] Y. Naito, T. Suzuki, Radial symmetry self- similar solutions for semilinear heat equations, J. Diff. Equ., 163 (2000), 407-428. 
[9] Y.W. Qi, The global existence and non uniqueness of a non linear degenerate equation, Nonlinear Analysis: Theory, Methods and Applications Journal, 31, No. 9 (1998), 117-136.

[10] E. Yanagida, Uniqueness of rapidly decaying solutions to the Haraux weissler equation, J. Diff. Equ., 127 (1996), 561-570. 
